\title{
Pancreas allocation and diabetic end-stage kidney disease
}

\author{
Nuno Moreira Fonseca \\ Nephrology Department, Centro Hospitalar Universitário de Lisboa Central
}

\section{ABSTRACT}

Transplant options for patients with type 1 diabetes and end-stage kidney disease include deceased donor kidney, live donor kidney, simultaneous pancreas-kidney, pancreas after kidney and islet cell transplantation. Allocation of deceased donor kidneys is prioritized for candidates of multiorgan transplants. Here, outcomes of treatment modalities are reviewed, as well as the ethical implications of multiorgan allocation of kidney-pancreas.

Keywords: end-stage kidney disease; diabetes; pancreas allocation; transplantation

\section{INTRODUCTION}

Historically, the only treatment for patients with type 1 diabetes mellitus (T1DM) was subcutaneous insulin injections. However, the use of daily insulin injections cannot exert the same degree of glycemia control, or completely alleviate the long-term vascular and neurological complications associated with T1DM. ${ }^{1}$ Since the first pancreas transplant at the University of Minnesota in 1966, whole-organ pancreas transplantation has become an established treatment for selected insulin-requiring diabetic patients with end-stage kidney disease (ESKD). ${ }^{2}$ While dialysis and insulin independence are the goal for all eligible ESKD TD1M patients, the transplant community has struggled for a long time to identify the best modality to achieve this while allocating scarce kidneys and pancreata in an equitable way. Here, available treatment modalities for ESKD TD1M and the ethical implications for multiorgan allocation are reviewed.

\section{PANCREAS TRANSPLANTATION FOR TYPE 2 DIABETES MELLITUS}

Pancreas transplantation has only been considered suitable for T1DM patients and there is uncertainty of its benefit for type 2 diabetes mellitus (T2DM) patients, due to impaired insulin action in target tissues in T2DM. ${ }^{3}$ However, the pathophysiology of T2DM is heterogeneous and some T2DM patients become euglycemic after pancreas transplantation. ${ }^{4}$ Therefore, for the purpose of the subsequent outcome analysis, only T1DM patients will be considered.

\section{TREATMENT OPTIONS FOR ESKD T1DM PATIENTS}

ESKD T1DM patients have at their disposal a wide array of treatment options, including dialysis; kidney transplantation alone, from either a live (LDKA) or a deceased donor (DDKA); kidney transplantation with whole-organ pancreas, which can be done simultaneously (SPK), or with a pancreas transplant after kidney of a deceased (DDK+PAK) or live donor (LDK+PAK); beta-cell replacement can also be done in the form of islet cell transplantation (ICT) after kidney transplantation of a deceased (DDK+ICT) or live donor (LDK+ICT), or simultaneously with a kidney transplant (SKI). ${ }^{5}$

The survival benefit of kidney transplantation is well established when compared to dialysis alone in diabetic nephropathy. ${ }^{6}$ While pancreas transplantation has gained momentum since it was first performed in 1966, the lack of randomized trials comparing its added benefit to kidney transplantation alone (KTA) partially curtails optimal decision making. ${ }^{2}$ The interpretation of currently available data is complex for several reasons. Evidence varies from single-center experience to analysis of large international registries. While the former may lack statistical significance, the latter may be hindered by heterogeneity of data quality, reflecting a myriad of immunosuppressive strategies. It is often difficult to judge whether patient groups receiving different transplantation strategies share an equal burden of cardiovascular risk, since information on severity of diabetes, cardiovascular morbidity or diabetic complications is often lacking. Most transplantation studies focus on patient survival and graft function outcomes. In addition to survival metrics, ponderation of treatment modality should also include consideration of the patient's preferences, as well as fitness for surgery, risk of hypoglycemic unawareness, progression of diabetic complications, and quality of life.

\section{Kidney Transplantation Alone}

For eligible candidates, kidney transplantation rather than remaining on dialysis is recommended. An analysis of the United States Renal Data System showed a projected increased lifespan of 11 years for diabetic patients who undergo transplantation compared to those who remain on dialysis. ${ }^{7}$ Generally LDKA recipients present better patient and graft survival rates than DDKA recipients. This survival benefit is also seen in diabetic recipients. ${ }^{8}$ Furthermore, LDKA allows for preemptive kidney transplant, thus avoiding the morbidity and increased cost of dialysis. 


\section{Kidney Transplantation with Pancreas Transplantation}

For T1DM ESKD patients able to withstand surgical complications, whole pancreas transplantation has become the gold standard treatment to achieve glycemic control and insulin independence. ${ }^{9}$ Pancreas transplantation can be performed simultaneously with a deceased kidney donor (SPK) or after a deceased or living donor (DDK+PAK or LDK+PAK). Both modalities of pancreas after kidney (PAK) transplant are associated with increased kidney graft survival compared with T1DM recipients who receive only a kidney. ${ }^{10} \mathrm{LDK}+\mathrm{PAK}$ present superior graft survival than DDK+PAK. ${ }^{10}$ Likewise, an analysis of the UK Transplant Registry on all SPK $(n=1739)$ and LDK $(n=385)$ transplants performed in the UK between 2001 and 2014 showed that SPK recipients with a functioning pancreas at 90 days present a better long-term patient survival and comparable kidney graft survival than LDK recipients. ${ }^{5}$ Thus, it seems that receiving a pancreas transplant may have a protective effect on the kidney allograft as glycemic control limits progression of microscopic lesions of diabetic nephropathy. ${ }^{11}$ Whether patients should undergo PAK or SPK remains a subject of debate. Interpretation of currently available data comparing outcomes of both modalities is hampered by variability of wait-list time for SPK, dialysis survival outcomes variability across different eras and countries, and the use of different immunosuppression regimens. In the setting of contradictory data, a recent analysis of the Organ Procurement and Transplantation Network (OPTN) data from 1995 to 2010 comprising over 12,000 SPK and 3,000 PAK transplants performed in the United States (US), has shown that SPK recipients presented a higher survival than PAK recipients, at 10 years post-transplant ( $70.3 \%$ vs $63.2 \%$ and $P<0.001) .1{ }^{10,12}$ While SPK remains, perhaps, the preferable transplant modality due to increased survival outcomes, if waiting time for an SPK is expected to be prolonged, LDK+PAK remains a reasonable approach, as it decreases waiting time and exposure to uremia, ${ }^{3,11-12}$

\section{Islet Cell Transplantation}

Pancreatic islet transplantation is a $\beta$-cell replacement therapy which normalizes glycemic control in T1DM patients. While not currently available in Portugal as a treatment option, it has been performed in over 1,500 patients worldwide. ${ }^{13}$ To date, there have been no randomized trials comparing the outcomes from whole organ versus islet transplantation. ICT is associated with lesser morbidity compared to whole organ transplantation, since the latter does require invasive surgery. Although it is a promising alternative, current outcomes remain suboptimal, showing an underperforming rate of insulin independence when compared to pancreas transplantation at 5 years $(73.6$ vs. $9.3 \%) .{ }^{14}$

\section{MULTIORGAN ALLOCATION}

In Portugal, kidney allocation follows multiorgan allocation, as all kidneys from deceased donors deemed appropriate are first offered to SPK recipients, except for peditric recipients and patients deemed urgent. ${ }^{15}$ This high degree of priority can potentially overwhelm the allocation system by redirecting all the "better" quality kidneys (if allocated with pancreata) to T1DM patients, thus placing all other potential recipients at a disadvantage. Therefore, the degree of priority ought to be proportional to both its need, i.e. the particular concern it addresses, as well as to the impact it produces in the remaining subgroups.

Firstly, when granting priority to a subset of patients, a sound ethical justification should be agreed upon, to avoid the risk of allocating organs in an unfair way. The established principles, or ethical theories, that support the current priorities in the Portuguese allocation system are as follows. Priority for peditric recipients is based on the "Prudential Lifespan Account", which claims that children with ESKD have a time-limited opportunity for growth and development and may endure lifelong consequences if not promptly transplanted. As a result, children have the potential to receive unique benefits that will positively affect them the entirety of their lives, thus justifying the need for priority..$^{16}$ In regard to patients deemed medically urgent, due to lack of a dialysis access, it is Jonsens" "Rule of Rescue" that grants legitimacy for current priority. According to this ethical rule, there is an imperative for people to rescue identifiable individuals facing avoidable death. In this case, granting utter priority to these patients avoids an extremely premature death, to which other candidates are not subject, even if they would also have a life-extending benefit. $^{17}$

What justifies granting priority for T1DM ESKD patients? T1DM patients are "worse off" than the remaining candidates in the sense that a simultaneous pancreas transplant allows for substantial benefits, including avoiding multiple surgeries, reduced risk of severe hypoglycemic episodes, and improved graft and patient survival, in opposition to single kidney transplant. Therefore, their claim for priority is strongly based on Rawls' "Difference Principle", which sustains that distributive justice is attained by maximising the interests of those deemed to be "worse off", when all other conditions remain equal. ${ }^{18}$

However, priority granting cannot be given with total disregard for the outcomes it produces. And while the previous argument may justify a higher degree of priority for SPK candidates over other candidates with similar or greater waiting list time, it is also necessary to evaluate the impact produced by preferential allocation of supposedly "better" kidneys to SPK recipients. In the US, SPK recipients are allocated grafts with a significantly lower average kidney donor profile index (KDPI) score - highest quality grafts - than kidney-alone recipients (18 vs 46). ${ }^{19}$ In our center, from 2011-2015, we have also seen a difference between average KPDI of SPK and single kidney grafts (30 vs 63). ${ }^{20}$ Lower KDPI for SPK is an expected result of stricter donor selection criteria. To evaluate the impact of this shift of lower KPDI grafts towards SPK recipients on other candidates, it is paramount to understand its relative proportion in kidney transplant activity overall. Of the 450 deceased donor kidney grafts performed in Portugal in 2017, 10 were allocated to peditric recipients (2\%) and 26 to SPK recipients $(6 \%) .{ }^{21}$ Preferential allocation of higher quality grafts remains marginal. Thus, priority of allocation to T1DM patients is ethically justifiable without hazarding unproportionally other patient subgroups. Extending SPK to selected T2DM patients will likely decrease the existing marginality of preferential allocation. Nevertheless, whatever impact it may have, it will also have to be pondered in relation to the unique benefits it can produce, which at this point are still not clear. In the US, the OPTN pancreas/kidney-pancreas allocation system in place since 2014 limits listing of T2DM ESDK patients 
(conveyed as patients with high C-peptide and high body mass index) for SPK so that these patients will not exceed $15 \%$ of the national list. There has been disagreement on the imposition of this limit. ${ }^{22}$ It is possible that, as the number of T2DM patients in the US increases, new evidence on outcomes will contribute to assessing the appropriateness of such limit.

\section{CONCLUSION}

While pancreas transplant is frequently regarded only as a lifeenhancing treatment, there is abundant evidence that successful pancreas transplant is clearly a life-extending procedure in addition to a kidney transplant. When reaching ESKD, selected T1DM should be offered both kidney and pancreas transplantation. If a prolonged waiting time is not a concern, SPK currently seems the best available option. However, if a living donor is available, and the expected waiting time is long, an LDK+PAK should be contemplated. The decision regarding treatment modality must also consider co-morbidities, fitness for surgery and patient preference. Allocation policies are subject to frequent review as new data supports the need for improvement. While SPK provides substantial clinical advantages to recipients these must be weighed against the claims and waiting times of other kidney candidates. Prioritizing a subgroup of recipients inexorably impacts other subgroups. The degree of priority should be proportional to both its need, i.e. the particular concern it addresses, as well as to the impact it produces in the remaining subgroups. Current allocation of deceased donor kidneys for SPK remains marginal in the Portuguese context.

Disclosure of potential conflicts of interest: Nuno Moreira Fonseca has received funding from Astellas, Fresenius Medical Care, Menarini, Sanofi ( participation in scientific meetings); AstraZeneca and Bial (participation in continuing medical education activities); GlaxoSmithKline and Bayer (clinical research); the Fulbright Commission (for postgraduate studies).

\section{References}

1. Ridgway, D. M., White, S. A., Kimber, R. M., \& Nicholson, M. L. Current practices of donor pan creas allocation in the UK: future implications for pancreas and islet transplantation. Transplant Int 2005; 18(7): 828-834

2. Squifflet, J. P., Gruessner, R. W., \& Sutherland, D. E. R. The history of pancreas transplantation: past, present and future. Acta Chir Belg 2008; 108(3): 367-378.

3. Cohen, D. J., \& Ratner, L. E. Type 2 diabetes: the best transplant option is still uncertain. Clin J Am Soc Neph 2012; 7(4): 530-532.

4. Hudson, A., L. Bradbury, R. Johnson, S. V. Fuggle, J. A. M. Shaw, J. J. Casey, P. J. Friend, and C. J. E. Watson. The UK pancreas allocation scheme for whole organ and islet transplantation. Am J Transplant; 2015; 15(9): 2443-2455.
5. Barlow, A. D., Saeb-Parsy, K., \& Watson, C. J. An analysis of the survival outcomes of simultaneous pancreas and kidney transplantation compared to live donor kidney transplantation in patients with type 1 diabetes: a UK Transplant Registry study. Transplant Int 2015; 30(9): 884-892.

6. Schnuelle, P., Lorenz, D., Trede, M., \& Van Der Woude, F. J. Impact of renal cadaveric transplantation on survival in end-stage renal failure: evidence for reduced mortality risk compared with hemodialysis during long-term follow-up. J Am Soc Neph 1998; 9(11): 2

7. Wolfe, R.A., Ashby, V.B., Milford, E.L., Ojo, A.O., Ettenger, R.E., Agodoa, L.Y., Held, P.J. and Port, F.K. Comparison of mortality in all patients on dialysis, patients on dialysis awaiting transplantation, and recipients of a first cadaveric transplant. New Eng J Med 1999; 341(23): 1725-1730.

8. Matas A.J., Smith J.M., Skeans M.A., Lamb K.E., Gustafson S.K., Samana C.J., Stewart D.E., Snyder J.J., Israni A.K., \& Kasiske B.L. OPTN/SRTR 2011 Annual Data Report: Kidney. Am J Transplant 2013; 13: $11-46$.

9. Fridell, J. A., \& Powelson, J. A. Pancreas after kidney transplantation: why is the most logical option the least popular?. Curr Op Organ Transp 2015; 20(1): 108-114.

10. Fridell, J. A., Niederhaus, S., Curry, M., Urban, R., Fox, A., \& Odorico, J. The survival advantage of pancreas after kidney transplant. Am J Transplant 2019; 19(3): 823-830.

11. Bilous, R.W., Mauer, S.M., Sutherland, D.E., Najarian, J.S., Goetz, F.C. and Steffes, M.W. The effects of pancreas transplantation on the glomerular structure of renal allografts in patients with insulin-dependent diabetes. New Eng J Med 1989; 321(2): 80-85.

12. Bazerbachi, F., Selzner, M., Marquez, M.A., Norgate, A., McGilvray, I.D., Schiff, J. and Cattral, M.S. Pancreas-after-kidney versus synchronous pancreas-kidney transplantation: comparison of intermediate-term results. Transplantation 2013; 95(3): 489-494.

13. Pérez-Sáez, M., \& Pascual, J. Kidney transplantation in the diabetic patient. J Clin Med 2015; 4(6): 1269-1280

14. Kleinclauss, F., Fauda, M., Sutherland, D.E., Kleinclauss, C., Gruessner, R.W., Matas, A.J., Kasiske, B.L., Humar, A., Kandaswamy, R., Kaul, S. and Gruessner, A.C. Pancreas after living donor kidney transplants in diabetic patients: impact on long-term kidney graft function. Clin Transplant 2009; 23(4): 437-446

15. Shapiro, A. J., Pokrywczynska, M., \& Ricordi, C. Clinical pancreatic islet transplantation. Nat Rev Endo 2017; 13(5): 268.

16. Lehmann, R., Graziano, J., Brockmann, J., Pfammatter, T., Kron, P., de Rougemont, O., Mueller, T., Zuellig, R.A., Spinas, G.A. and Gerber, P.A. Glycemic control in simultaneous islet-kidney versus pancreas-kidney transplantation in type 1 diabetes: a prospective 13-year follow-up. Diabetes Care 2015; 38(5): 752-759.

17. Circular normativa N.a 15/GDC de 07/09/2009, 7 (2018, November 15). Retrieved from: http:// www.ipst.pt/

18. Daniels, N. Am I my parents' keeper?: an essay on justice between the young and the old (Vol. 10). New York: Oxford University Press 1998.

19. McKie, J., \& Richardson, J. The rule of rescue. Social science \& medicine 2003; 56(12): 2407-2419.

20. Rawls, J. A theory of justice. 1971; 132-80.

21. Reese, P. P., Veatch, R. M., Abt, P. L., \& Amaral, S. Revisiting multi-organ transplantation in the setting of scarcity. Am J Transplant 2014; 14(1): 21-26.

22. Moreira Fonseca, N., Silva, C., Cotovio, P., Caeiro, F., Aires, I., Possante, M., Nolasco, F. Análise da Utilidade no Sistema Português de Alocação de Rins Cadáver. Abstract presented to XIII Congresso Português de Transplantação, XV Congresso Luso Brasileiro de Transplantação e II Encontro Ibérico de Transplantação; Porto, Portugal; October 2016.

23. Portuguese Registry of Dialysis and Transplantation 2017 (2018, November 15). Retrieved from: http://www.spnefro.pt/t

24. Al-Qaoud, T. M., Odorico, J. S., \& Redfield III, R. R. Pancreas transplantation in type 2 diabetes: expanding the criteria. Curr op organ transplant 2018; 23(4): 454-460.

\section{Correspondence to:}

Nuno Moreira Fonseca, MD, MA

Nephrology Department, Centro Hospitalar Universitário de Lisboa Central, Lisboa, Portugal

E-mail: nuno.mf@nyu.edu 